\title{
Application of Modified Adomian Decomposition Method to (2+1)- dimensional Non-linear Wu-Zhang system
}

\author{
Ahmed F. Qasim \\ College of Computer Sciences and \\ Mathematics \\ University of Mosul \\ Republic of Iraq \\ E-mail: ahmednumerical@yahoo.com
}

Zead Y. Ali

College of physical education and sport scinces

University of Mosul

Republic of Iraq

E-mail: zyaa_77@yahoo.com

Recived : 2611112017

Revised : //

Accepted : 14\12\2017

Available online : $\quad 22 / 1 / 2018$

DOI: $10.29304 /$ jqcm.2018.10.1.340

\begin{abstract}
In this paper, the Domain decomposition method (ADM) with modified polynomials is applied for nonlinear $(2+1)$ - dimensional Wu-Zhang system . we compared the solution of the system with MVIM, HPM and RDTM [ 17,13,15]. The numerical results obtained by this polynomial are very effective, convenient and quite accurate to system of partial differential equations. A comparative between the modifications method and the other methods is present from some examples to show the efficiency of each method.
\end{abstract}

Keywords: Modified Adomian decomposition method, Wu- Zhang system.

Mathematics Subject Classification: 65M99,35G50 .

\section{Introduction}

The nonlinear partial differential equations (NPDEs) are encountered in various disciplines, such as physics, mechanics, chemistry, biology, mathematics and engineering. Many efforts have been made on the study of NPDEs. Long wave in shallow water is a subject of broad interests and has along colorful history.
Wu and Zhang derived three sets of model equations for modeling nonlinear and dispersive long gravity waves travelling in two horizontal directions on shallow waters of uniform depth [16]. One of these equations, Wu-Zhang equation (which describes $(2+1)$-dimensional dispersive long wave), can be written as[16] 


$$
\begin{aligned}
& u_{t}+u u_{x}+v u_{y}+w_{x}=0, \\
& v_{t}+u v_{x}+v v_{y}+w_{y}=0,(1) \\
& w_{t}+(u w)_{x}+(v w)_{y}+ \\
& \quad \quad \frac{1}{3}\left(u_{x x x}+u_{x y y}+u_{x x y}+u_{y y y}\right)=0,
\end{aligned}
$$

where $\mathrm{w}$ is the elevation of the water, $\mathrm{u}$ is the surface velocity of water along $\mathrm{x}$-direction, and $\mathrm{V}$ is the surface velocity of water along y-direction. The explicit solutions of Eq. (1) are very helpful for costal and civil engineers to apply the nonlinear water wave model in harbor and coastal design. Recently introduced some new methods and are applied to nonlinear equations such as Variation Iteration Method [11], Homotopy Perturbation Method [10,13], the homogeneous balance method [9], the differential transform method (DTM) [5]. Among all of the methods, the reduced differential transform method (RDTM), employed for solving the nonlinear partial differential equations [12]. The RDTM is presented to overcome the demerit of complex calculation of DTM. This technique doesn't require any discretization, linearization or small perturbations and therefore it reduces significantly the numerical computation.

Ma Z.Y [13], used Homotopy perturbation method (HPM) for the Wu-Zhang equation in fluid dynamics. Extended tanh method and expfunction method and its application to $(2+1)$ dimensional dispersive long wave nonlinear equations [4]. Application to Reduced differential Transform method to the Wu-Zhang equation is presented in [15], and the solution of $\mathrm{Wu}$-Zhang equations using the modified variational iteration method obtained by [17]. solitons and other solution Wu-Zhang system are discussed in [14].
At the beginning of the 1980, a so-called Adomian decomposition method (ADM) [1] has been used to solve effectively, easily, and accurately a large class of linear and nonlinear equations, solutions partial, deterministic or stochastic differential equations with approximates which converge rapidly.

Several studies have been proposed to modify the regular Adomian decomposition method for initial value problems in ordinary and partial differential equations [3,6]. Error analysis of Adomian series solution to a class of nonlinear differential equation is introduction in [7]. Alkresheh ,H. A. [2] , proposed two new adomian decomposition method, and [8] used the ADM to solve coupled system of nonlinear physical equations, coupled system of diffusion-reaction equation and integro-differential diffusion-reaction equation.

This paper is organized as follows. In section 2, the basic ideas of the modified Adomian decomposition method are described, in section 3 , is devoted to solving a nonlinear $(2+1)$ dimensional Wu-Zhang system by MADM, the results and comparisons of

the numerical solutions are presented in section 4 and concluding remarks are given in section 5 . 


\section{The modified decomposition (MADM)} equation

Let us consider the following

$L u+N u+R u=f(x)$

where $L$ is an invertible linear operator, $N$ represents the nonlinear operator and $R$ is the remaining linear part, from equation (2) we have

$L u=f(x)-N u-R u$,

Now, applying the inverse operator $L^{-1}$ to both sides of equation (3) then use the initial conditions we find

$$
u=g(x)-L^{-1} N u-L^{-1} R u,(4)
$$

where $L^{-1}=\int_{0}^{x}() \quad d$.$s , and \mathrm{g}(x)$ represents the terms having from integrating the remaining term $f(x)$ and from using the given initial or boundary conditions. The ADM assumes that the nonlinear operator $N(u)$ can be decomposed by an infinite series of polynomials given by

$N(u)=\sum_{n=0}^{\infty} A_{n}\left(u_{o}, u_{1}, \ldots, u_{n}\right)$ where $A_{\mathrm{n}}$ are the Adomian's polynomials [1] defined as:

$$
\begin{aligned}
& A_{n}= \\
& \frac{1}{n !} \frac{d^{n}}{d \lambda^{n}}\left[N\left(\sum_{i=0}^{\infty} \lambda^{\mathrm{i}} \mathrm{u}_{\mathrm{i}}\right)\right]_{\lambda=0} \\
& , \quad \mathrm{n}=0,1,2, \ldots
\end{aligned}
$$

El-Kalla [7] introduce a new formula for Adomian polynomials, he claimed that the Adomian solution using this new formula converges faster than using Adomian polynomials (5). Kalla polynomial given in the following form:

$$
\begin{aligned}
& A_{n}= \\
& \quad N\left(S_{n}\right)- \\
& \sum_{i=0}^{n-1} A_{i}\left(u_{o}, u_{1}, \ldots, u_{n-1}\right)
\end{aligned}
$$

Where $S_{n}=u_{o}+u_{1}+\cdots+u_{n} \quad$ and $A_{\mathrm{n}}$ can be given as:

$$
\begin{aligned}
& A_{0}=N\left(u_{o}\right) \\
& A_{1}=\quad \frac{d}{d x}\left(N\left(u_{o}\right)\right) u_{1}+ \\
& \quad \frac{1}{2} \frac{d^{2}}{d x^{2}}\left(N\left(u_{o}\right)\right) u_{1}^{2}+ \\
& \quad \frac{1}{6} \frac{d^{3}}{d x^{3}}\left(N\left(u_{o}\right)\right) u_{1}^{3}+ \\
& \quad \frac{1}{24} \frac{d^{4}}{d x^{4}}\left(N\left(u_{o}\right)\right) u_{1}^{4}+\cdots \\
& A_{2}=\frac{d}{d x}\left(N\left(u_{o}\right)\right) u_{2}+ \\
& \quad \frac{1}{2} \frac{d^{2}}{d x^{2}}\left(N\left(u_{o}\right)\right)\left[2 u_{1} u_{2}+u_{2}^{2}\right]+ \\
& \quad \frac{1}{6} \frac{d^{3}}{d x^{3}}\left(N\left(u_{o}\right)\right)\left[3 u_{1}^{2} u_{2}+\right. \\
& \left.\quad 3 u_{1} u_{2}^{2}+u_{2}^{3}\right]+\cdots
\end{aligned}
$$

And so on. These formulas are easy to compute by using Maple 13 software.

\section{Application MADM to the Wu- Zhang system}

This section is devoted to study the analytical solution of the $\mathrm{Wu}$ Zhang system (1) using MADM, we make a comparison for the corresponding absolution error between the using of the proposed polynomial in MADM and the methods in $[13,15,17]$.

let us consider the standard form of equation (1) in an operator form:

$$
\begin{gathered}
L_{t} u+u L_{x} u+v L_{y} u+L_{x} w=0, \\
L_{t} v+u L_{x} v+v L_{y} v+L_{y} w=0,(7) \\
L_{t} w+u L_{x} u+w L_{x} u+v L_{y} w+ \\
w L_{y} v+\frac{1}{3}\left(L_{x x x} u+L_{x y y} u+\right. \\
\left.L_{x x y} v+L_{y y y} v\right)=0, \\
u(x, y, o)=g_{1}(x, y), v(x, y, o)= \\
g_{2}(x, y), w(x, y, o)=g_{3}(x, y),
\end{gathered}
$$

Where the notations $L_{t}=\frac{\partial}{\partial_{t}}, L_{x}=$ $\frac{\partial}{\partial_{x}}, L_{y}=\frac{\partial}{\partial_{y}}, L_{x x x}=\frac{\partial^{3}}{\partial_{x^{3}}}, L_{x y y}=$ $\frac{\partial^{3}}{\partial_{x} \partial_{y^{2}}}, L_{x x y}=\frac{\partial^{3}}{\partial_{x^{2}} \partial_{y}}$, and $L_{y y y}=\frac{\partial^{3}}{\partial y^{3}}$, Symbolize the linear differential operator. 
Assuming $l_{t}^{-1}$ the inverse of the operator $l_{t}$ exists and can conveniently by taken as definite integrate with respect to $(\mathrm{t})$ :

Define $l_{t}^{-1}=\int_{0}^{t}() d$.$t than the system$

(7) becomes :

$$
\begin{aligned}
u(x, y, t)= & g_{1}(x, y)-l_{t}^{-1}\left(\vartheta_{1}(u)+\right. \\
& \left.\vartheta_{2}(u, v)+l_{x} w\right), \\
v(x, y, t)= & g_{2}(x, y)-l_{t}^{-1}\left(\vartheta_{3}(u, v)+\right. \\
& \left.\vartheta_{4}(v)+l_{y} w\right), \\
w(x, y, t)= & g_{3}(x, y)- \\
& l_{t}^{-1}\left(\vartheta_{5}(u, w)+\right. \\
& \vartheta_{6}(u, w)+\vartheta_{7}(v, w)+ \\
& \vartheta_{8}(v, w)+ \\
& \frac{1}{3}\left(L_{x x x} u+L_{x y y} u+\right. \\
& \left.\left.L_{x x y} v+L_{y y y} v\right)\right),
\end{aligned}
$$

Where

$$
\begin{aligned}
& \vartheta_{1}(u)=u u_{x}, \vartheta_{2}(u, v)= \\
& v u_{y}, \vartheta_{3}(u, v)=u v_{x} \\
& \vartheta_{4}(v)=v v_{x}, \vartheta_{5}(u, w)= \\
& u w_{x}, \vartheta_{6}(u, w)=w u_{x} \\
& \vartheta_{7}(v, w)=v w_{y}, \vartheta_{8}(u, w)=w v_{y}
\end{aligned}
$$

The MADM assumes an infinite series for the unknown functions $u(x, y, t), v(x, y, t)$ and $w(x, y, t)$

in the form

$$
\begin{aligned}
& u(x, y, t)=\sum_{n=0}^{\infty} u_{n}(x, y, t) \\
& v(x, y, t)=\sum_{n=0}^{\infty} v_{n}(x, y, t) \\
& w(x, y, t)=\sum_{n=0}^{\infty} w_{n}(x, y, t)
\end{aligned}
$$

We can write $\vartheta_{1}, \vartheta_{2} \ldots \ldots \ldots, \vartheta_{8}$ by an infinite series of Adomian polynomial in the form

$\vartheta_{1}(u)=\sum_{n=0}^{\infty} A_{n} \quad, \vartheta_{2}(u, v)=$ $\sum_{n=0}^{\infty} B_{n}, \vartheta_{3}(u, v)=\sum_{n=0}^{\infty} C_{n}$ $\vartheta_{4}(v)=\sum_{n=0}^{\infty} D_{n}, \vartheta_{5}(u, w)=$ $\sum_{n=0}^{\infty} E_{n}, \vartheta_{6}(u, w)=\sum_{n=0}^{\infty} F_{n}$ $\vartheta_{7}(v, w)=\sum_{n=0}^{\infty} G_{n} \quad, \vartheta_{8}(v, w)=$ $\sum_{n=0}^{\infty} H_{n}$

Where $A_{n}, B_{n}, C_{n}, D_{n} \ldots \ldots, H_{n}$ are the appropriate modified Adomian polynomials

$$
\begin{aligned}
& A_{n}\left(u_{0}, u_{1}, \ldots \ldots \ldots . u_{n}\right)= \\
& \frac{1}{n !} \frac{d^{n}}{d \rho^{n}}\left[\varphi_{1}\left(\sum_{k=0}^{\infty} \rho^{k} u_{k}\right)\right]_{\rho=0}, n \geq 0 \\
& B_{n}\left(u_{0}, u_{1}, \ldots, u_{n}, v_{0}, v_{1}, \ldots, v_{n}\right) \\
& =\frac{1}{n !} \frac{d^{n}}{d \rho^{n}}\left[\varphi_{2}\left(\sum_{k=0}^{\infty} \rho^{k} u_{k}, \sum_{k=0}^{\infty} \rho^{k} v_{k}\right)\right]_{\rho=0} \\
& \quad, n \geq 0 \\
& \vdots \quad(8) \quad \\
& H_{n}\left(v_{0}, v_{1}, \ldots, v_{n}, w_{0}, w_{1}, \ldots, w_{n}\right)= \\
& \frac{1}{n !} \frac{d^{n}}{d \rho^{n}}\left[\varphi_{8}\left(\sum_{k=0}^{\infty} \rho^{k} v_{k}, \sum_{k=0}^{\infty} \rho^{k} w_{k}\right)\right]_{\rho=0}, \\
& n \geq 0
\end{aligned}
$$

For examples, the first polynomials using formulas (8) are computed be:

$$
\begin{aligned}
& A_{o}=u_{o} \frac{\partial u_{o}}{\partial x} \\
& A_{1}=u_{o} \frac{\partial u_{1}}{\partial x}+u_{1} \frac{\partial u_{o}}{\partial x}+u_{1} \frac{\partial u_{1}}{\partial x} \\
& A_{2}=u_{o} \frac{\partial u_{2}}{\partial x}+u_{2} \frac{\partial u_{o}}{\partial x}+u_{2} \frac{\partial u_{1}}{\partial x}+ \\
& u_{1} \frac{\partial u_{2}}{\partial x}+u_{2} \frac{\partial u_{2}}{\partial x} \\
& A_{3}=u_{3} \frac{\partial u_{o}}{\partial x}+u_{o} \frac{\partial u_{3}}{\partial x}+u_{3} \frac{\partial u_{1}}{\partial x}+ \\
& u_{1} \frac{\partial u_{3}}{\partial x}+\cdots \\
& A_{4}=u_{4} \frac{\partial u_{o}}{\partial x}+u_{o} \frac{\partial u_{4}}{\partial x}+\cdots \\
& \vdots \\
& B_{o}=v_{o} \frac{\partial u_{o}}{\partial y} \\
& B_{1}=v_{o} \frac{\partial u_{1}}{\partial y}+v_{1} \frac{\partial u_{o}}{\partial y}+v_{1} \frac{\partial u_{1}}{\partial y} \\
& B_{2}=v_{o} \frac{\partial u_{2}}{\partial y}+v_{2} \frac{\partial u_{o}}{\partial y}+v_{2} \frac{\partial u_{1}}{\partial y}+ \\
& v_{1} \frac{\partial u_{2}}{\partial y}+v_{2} \frac{\partial u_{2}}{\partial y} \\
& B_{3}=v_{3} \frac{\partial u_{o}}{\partial y}+v_{o} \frac{\partial u_{3}}{\partial y}+v_{3} \frac{\partial u_{1}}{\partial y}+ \\
& v_{1} \frac{\partial u_{3}}{\partial y}+\cdots \\
& C_{o}=u_{o} \frac{\partial v_{o}}{\partial x} \\
& C_{1}=u_{o} \frac{\partial v_{1}}{\partial x}+u_{1} \frac{\partial v_{0}}{\partial x}+u_{1} \frac{\partial v_{1}}{\partial x} \\
& C_{2}=u_{o} \frac{\partial v_{2}}{\partial x}+u_{2} \frac{\partial v_{0}}{\partial x}+u_{2} \frac{\partial v_{1}}{\partial x}+ \\
& u_{1} \frac{\partial v_{2}}{\partial x}+u_{2} \frac{\partial v_{2}}{\partial x} \\
& C_{3}=u_{3} \frac{\partial v_{0}}{\partial x}+u_{o} \frac{\partial v_{3}}{\partial x}+u_{3} \frac{\partial v_{1}}{\partial x}+ \\
& u_{1} \frac{\partial v_{3}}{\partial x}+\cdots
\end{aligned}
$$


Ahmed .F/ Zead .Y

$$
\vdots
$$$$
D_{o}=v_{o} \frac{\partial v_{o}}{\partial y}
$$$$
D_{1}=v_{o} \frac{\partial v_{1}}{\partial y}+v_{1} \frac{\partial v_{o}}{\partial y}+v_{1} \frac{\partial v_{1}}{\partial y}
$$$$
D_{2}=v_{o} \frac{\partial v_{2}}{\partial y}+v_{2} \frac{\partial v_{o}}{\partial y}+v_{2} \frac{\partial v_{1}}{\partial y}+
$$$$
v_{1} \frac{\partial v_{2}}{\partial y}+v_{2} \frac{\partial v_{2}}{\partial y}
$$$$
D_{3}=v_{3} \frac{\partial v_{o}}{\partial y}+v_{o} \frac{\partial v_{3}}{\partial y}+v_{3} \frac{\partial v_{1}}{\partial y}+
$$$$
v_{1} \frac{\partial v_{3}}{\partial y}+\cdots
$$

$$
\begin{aligned}
E_{o}= & u_{o} \frac{\partial w_{o}}{\partial x} \\
E_{1}= & u_{o} \frac{\partial w_{1}}{\partial x}+u_{1} \frac{\partial w_{o}}{\partial x}+u_{1} \frac{\partial w_{1}}{\partial x} \\
E_{2}= & u_{o} \frac{\partial w_{2}}{\partial x}+u_{2} \frac{\partial w_{o}}{\partial x}+u_{2} \frac{\partial w_{1}}{\partial x}+ \\
& u_{1} \frac{\partial w_{2}}{\partial x}+u_{2} \frac{\partial w_{2}}{\partial x} \\
E_{3}= & u_{3} \frac{\partial w_{o}}{\partial x}+u_{o} \frac{\partial w_{3}}{\partial x}+u_{3} \frac{\partial w_{1}}{\partial x}+ \\
& u_{1} \frac{\partial w_{3}}{\partial x}+\cdots
\end{aligned}
$$$$
F_{o}=w_{o} \frac{\partial u_{o}}{\partial x}
$$$$
F_{1}=w_{o} \frac{\partial u_{1}}{\partial x}+w_{1} \frac{\partial u_{o}}{\partial x}+w_{1} \frac{\partial u_{1}}{\partial x}
$$$$
F_{2}=w_{o} \frac{\partial u_{2}}{\partial x}+w_{2} \frac{\partial u_{o}}{\partial x}+w_{2} \frac{\partial u_{1}}{\partial x}+
$$$$
w_{1} \frac{\partial u_{2}}{\partial x}+w_{2} \frac{\partial u_{2}}{\partial x}
$$$$
F_{3}=w_{3} \frac{\partial u_{o}}{\partial x}+w_{o} \frac{\partial u_{3}}{\partial x}+w_{3} \frac{\partial u_{1}}{\partial x}+
$$$$
w_{1} \frac{\partial u_{3}}{\partial x}+\cdots
$$$$
\vdots
$$$$
G_{o}=v_{o} \frac{\partial w_{o}}{\partial y}
$$$$
G_{1}=v_{o} \frac{\partial w_{1}}{\partial y}+v_{1} \frac{\partial w_{o}}{\partial y}+v_{1} \frac{\partial w_{1}}{\partial y}
$$$$
G_{2}=v_{o} \frac{\partial w_{2}}{\partial y}+v_{2} \frac{\partial w_{o}}{\partial y}+v_{2} \frac{\partial w_{1}}{\partial y}+
$$$$
v_{1} \frac{\partial w_{2}}{\partial y}+v_{2} \frac{\partial w_{2}}{\partial y}
$$$$
G_{3}=v_{3} \frac{\partial w_{o}}{\partial y}+v_{o} \frac{\partial w_{3}}{\partial y}+v_{3} \frac{\partial w_{1}}{\partial y}+
$$$$
v_{1} \frac{\partial w_{3}}{\partial y}+\cdots
$$$$
\begin{aligned}
H_{o}= & w_{o} \frac{\partial v_{o}}{\partial y} \\
H_{1}= & w_{o} \frac{\partial v_{1}}{\partial y}+w_{1} \frac{\partial v_{o}}{\partial y}+w_{1} \frac{\partial v_{1}}{\partial y} \\
H_{2}= & w_{o} \frac{\partial v_{2}}{\partial y}+w_{2} \frac{\partial v_{o}}{\partial y}+w_{2} \frac{\partial v_{1}}{\partial y}+ \\
& w_{1} \frac{\partial v_{2}}{\partial y}+w_{2} \frac{\partial v_{2}}{\partial y} \\
H_{3}= & w_{3} \frac{\partial v_{o}}{\partial y}+w_{o} \frac{\partial v_{3}}{\partial y}+w_{3} \frac{\partial v_{1}}{\partial y}+ \\
& w_{1} \frac{\partial v_{3}}{\partial y}+\cdots \\
\vdots &
\end{aligned}
$$

And so on, the nonlinear system (7) is constructed as:

$$
\begin{aligned}
& u_{0}(x, y, t)=g_{1}(x, y), v_{0}(x, y, t)= \\
& g_{2}(x, y), w_{0}(x, y, t)=g_{3}(x, y) \text {, } \\
& u_{n+1}(x, y, t)=l_{t}^{-1}\left[l_{x} w+A_{n}+B_{n}\right] \\
& n \geq 1 \\
& v_{n+1}(x, y, t)=l_{t}^{-1}\left[l_{y} w+C_{n}+D_{n}\right] \text {; } \\
& n \geq 1 \text {, (9) } \\
& w_{n+1}(x, y, t)=l_{t}^{-1}\left[\frac { 1 } { 3 } \left(L_{x x x} u\right.\right. \\
& +L_{x y y} u+L_{x x y} v \\
& \left.+L_{y y y} v\right)+E_{n}+F_{n} \\
& \left.+G_{n}+H_{n}\right] \text {; } \\
& n \geq 1
\end{aligned}
$$

Where The functions $g_{1}(x, y), g_{2}(x, y)$, and $g_{3}(x, y)$, Are initial conditions. We construct the solution $u(x, y, t), v(x, y, t)$ And $w(x, y, t)$ as follow:

$\lim _{n \rightarrow \infty} \varphi_{n}=u(x, y, t)$,

$\lim _{n \rightarrow \infty} \widetilde{\varphi_{n}}=v(x, y, t)$

and

$\lim _{n \rightarrow \infty} \widehat{\varphi_{n}}=w(x, y, t)$

$\varphi_{n}(x, y, t)=\sum_{k=0}^{\infty} u_{k}(x, y, t)$,

$\widetilde{\varphi_{n}}(x, y, t)=\sum_{k=0}^{\infty} v_{k}(x, y, t)$,

$\widehat{\varphi_{n}}(x, y, t)=\sum_{k=0}^{\infty} w_{k}(x, y, t)$

And the recurrence relation is given as in (9). 


\section{Applications}

We consider the Solutions of the system (9) with the initial and conditions $[13,15,17]$.

$$
\begin{aligned}
& u_{o}(x, y, t)=-\frac{K_{3}+K_{2} b b_{0}}{K_{1}}+ \\
& v_{o}(x, y, t)= \frac{2}{3} \sqrt{3} k_{1} \tanh \left(k_{1} x+k_{2} y\right), \\
& \frac{2}{3} \sqrt{3} k_{2} \tanh \left(k_{1} x+k_{2} y\right),
\end{aligned}
$$

$w_{o}(x, y, t)=\frac{2}{3}\left(k_{1}^{2}+k_{2}^{2}\right) \operatorname{sech}\left(k_{1} x+\right.$

Where

$$
\left.k_{2} y\right)^{2}
$$

$b_{0}, k_{1}, k_{2}$ and $k_{3}$ are arbitrary constants .

To calculate the terms of the MADN for $u(x, y, t), v(x, y, t)$ and $w(x, y, t)$, we substitute the initial conditions (10) into the system (9) and using Maple 13 language, the solutions of the system (9) can be obtained as follows:

$$
\begin{aligned}
& u_{1}(x, y, t)=\frac{2}{3} \frac{K_{1} t \sqrt{3} K_{3}}{\cosh \left(K_{1} x+K_{2} y\right)^{2}} \\
& v_{1}(x, y, t)=\frac{2}{3} \frac{K_{2} t \sqrt{3} K_{3}}{\cosh \left(K_{1} x+K_{2} y\right)^{2}} \\
& w_{1}(x, y, t)= \\
& -\frac{4}{3} \frac{t \sinh \left(K_{1} x+K_{2} y\right) K_{3}\left(K_{1}^{2} x+K_{2}^{2} y\right)}{\cosh \left(K_{1} x+K_{2} y\right)^{3}} \\
& u_{2}(x, y, t)= \\
& -\frac{2}{9} \frac{1}{\cosh \left(K_{1} x+K_{2} y\right)^{5}}\left(k _ { 1 } k _ { 3 } ^ { 2 } t ^ { 2 } \operatorname { s i n h } \left(k_{1} x+\right.\right. \\
& \left.k_{2} y\right)^{\left.\left.-4 K_{1}^{2} t-4 t K_{2}^{2}+3 \sqrt{3} \cosh \left(k_{1} x+k_{2} y\right)^{2}\right)\right)} \\
& v_{2}(x, y, t)= \\
& -\frac{2}{9} \frac{1}{\cosh \left(K_{1} x+K_{2} y\right)^{5}}\left(k _ { 1 } k _ { 3 } ^ { 2 } t ^ { 2 } \operatorname { s i n h } \left(k_{1} x+\right.\right. \\
& \left.k_{2} y\right)^{\left.\left.-4 K_{1}^{2} t-4 t K_{2}^{2}+3 \sqrt{3} \cosh \left(k_{1} x+k_{2} y\right)^{2}\right)\right)} \\
& w_{2}(x, y, t) \\
& =-\frac{2}{27} \frac{1}{\cosh \left(K_{1} x+K_{2} y\right)^{5}}\left(K_{3}^{2} t^{2}\right.
\end{aligned}
$$

$$
\begin{aligned}
& \left(-20 k_{1}^{4} t \sqrt{3}-40 k_{2}^{2} k_{1}^{2} \mathrm{t} \sqrt{3}-\right. \\
& 20 k_{2}^{4} \mathrm{t} \sqrt{3}+27 k_{1}^{2} \cosh \left(k_{1} x+\right. \\
& \left.k_{2} y\right)^{2}+27 k_{2}^{2} \cosh \left(k_{1} x+k_{2} y\right)^{2}- \\
& 18 k_{1}^{2} \cosh \left(k_{1} x+k_{2} y\right)^{4}- \\
& 18 k_{2}^{2} \cosh \left(k_{1} x+k_{2} y\right)^{4}+ \\
& 16 \sqrt{3} \mathrm{t} k_{1}^{4} \cosh \left(k_{1} x+k_{2} y\right)^{2}+ \\
& 16 \sqrt{3} \mathrm{t} k_{2}^{4} \cosh \left(k_{1} x+k_{2} y\right)^{2}+ \\
& \left.\left.32 \sqrt{3} \mathrm{t} k_{1}^{2} k_{1}^{2} \cosh \left(k_{1} x+k_{2} y\right)^{2}\right)\right) \\
& u(x, y, t)=u_{o}(x, y, t)+u_{1}(x, y, t)+ \\
& u_{2}(x, y, t)+\cdots \\
& \frac{-1}{2835} \frac{1}{k_{1} \cosh \left(k_{1} x+k_{2} y\right)^{11}}\left(2 8 3 5 \operatorname { c o s h } \left(k_{1} x+\right.\right. \\
& \left.k_{2} y\right)^{11} k_{2} b b_{0} \ldots \ldots \\
& v(x, y, t)=v_{o}(x, y, t)+v_{1}(x, y, t)+ \\
& v_{2}(x, y, t)+\cdots \\
& = \\
& \frac{1}{2835} \frac{1}{\cosh \left(k_{1} x+k_{2} y\right)^{11}}\left(2 8 3 5 b b _ { 0 } \operatorname { c o s h } \left(k_{1} x+\right.\right. \\
& \left.k_{2} y\right)^{11}-1890 \ldots \\
& w(x, y, t)=w_{o}(x, y, t)+ \\
& w_{1}(x, y, t)+ \\
& w_{2}(x, y, t)+\cdots \\
& = \\
& \frac{2}{8505} \frac{1}{\cosh \left(k_{1} x+k_{2} y\right)^{12}}\left(5 5 0 4 0 k _ { 3 } ^ { 4 } t ^ { 7 } \sqrt { 3 } k _ { 2 } ^ { 2 } k _ { 1 } ^ { 6 } \operatorname { c o s h } \left(k_{1} x+\right.\right. \\
& \left.k_{2} y\right)^{2}-20480 \ldots
\end{aligned}
$$

The approximate solutions of the system (1) are obtained as follows

$$
\begin{aligned}
u(x, y, t)= & u_{0}(x, y, t)+u_{1}(x, y, t)+ \\
& u_{2}(x, y, t)+\cdots \\
v(x, y, t)= & v_{0}(x, y, t)+v_{1}(x, y, t)+ \\
& v_{2}(x, y, t)+\cdots \\
w(x, y, t)= & w_{0}(x, y, t)+ \\
& w_{1}(x, y, t)+ \\
& w_{2}(x, y, t)+\cdots
\end{aligned}
$$

This solution is convergent to the exact solution [13]

$$
\begin{aligned}
u(x, y, t)= & -\frac{K_{3}+K_{2} b_{0}}{K_{1}}+ \\
& \frac{2 \sqrt{3}}{3} k_{1} \tanh \left(k_{1} x+\right. \\
& \left.k_{2} y+k_{3} t\right),
\end{aligned}
$$




$$
\begin{aligned}
v(x, y, t)= & b_{0}+\frac{2 \sqrt{3}}{3} k_{2} \tanh \left(k_{1} x+\right. \\
& \left.k_{2} y+k_{3} t\right), \\
w(x, y, t)= & \frac{2}{3}\left(k_{1}^{2}+k_{2}^{2}\right) \operatorname{sech}^{2}\left(k_{1} x+\right. \\
& \left.k_{2} y+k_{3} t\right),
\end{aligned}
$$

We comparisons the absolute errors and mean square error for the MADN results for $u(x, y, t), v(x, y, t)$ and $w(x, y, t)$, for the first three approximations with HPM [13] ,RDTM [15], and MVIM [17], when

$b_{0}=k_{1}=0.1, k_{2}=k_{3}=0.01$, $t=5, y=20$

for the solution of the Wu Zhang system (1) with the initial conditions (10).

Table 1: Comparison of absolute errors using $u_{3}$ for various values of $x$ in Wu-Zhang system between MADM and MVIM [17] when

$$
b_{0}=k_{1}=0.1, k_{2}=k_{3}=0.01, t=5, \text { and } y=20
$$

\begin{tabular}{|c|c|c|c|c|}
\hline$x$ & $u_{\text {exact }}$ & $u_{M A D M}$ & $\left|u_{\text {exact }}-u_{M V I M}\right|$ & $\left|u_{\text {exact }}-u_{\text {MADM }}\right|$ \\
\hline-50 & 0.225452768 & 0.225452769 & $1.6451825 \mathrm{E}-6$ & $10.39242830 \mathrm{E}-10$ \\
\hline-40 & 0.225342395 & 0.225342395 & $1.2148126 \mathrm{E}-5$ & $4.330797530 \mathrm{E}-10$ \\
\hline-30 & 0.224530095 & 0.224530099 & $8.9315718 \mathrm{E}-5$ & $3.594419403 \mathrm{E}-9$ \\
\hline-20 & 0.218700684 & 0.218700708 & $6.3605735 \mathrm{E}-4$ & $2.448538578 \mathrm{E}-8$ \\
\hline-10 & 0.183340683 & 0.183340786 & $3.6024380 \mathrm{E}-3$ & $1.023749896 \mathrm{E}-7$ \\
\hline 0 & 0.0817192288 & 0.0817189240 & $6.4447985 \mathrm{E}-3$ & $3.048061683 \mathrm{E}-7$ \\
\hline 10 & 0.0120486423 & 0.120487416 & $1.9107842 \mathrm{E}-4$ & $8.922341521 \mathrm{E}-3$ \\
\hline 20 & 0.002932728 & 0.002932733 & $2.9196656 \mathrm{E}-4$ & $5.192973059 \mathrm{E}-9$ \\
\hline 30 & 0.005123370 & 0.005123372 & $4.0172038 \mathrm{E}-5$ & $1.372972068 \mathrm{E}-9$ \\
\hline 40 & 0.005423074 & 0.005423074 & $5.44887493 \mathrm{E}-6$ & $2.078596792 \mathrm{E}-10$ \\
\hline 50 & 0.005463694 & 0.005463694 & $7.37648418 \mathrm{E}-7$ & $5.196177359 \mathrm{E}-11$ \\
\hline MSE & & & $5.00446211 \mathrm{E}-6$ & $1.018121171 \mathrm{E}-14$ \\
\hline
\end{tabular}


Table 2: Comparison of absolute errors using $v_{3}$ for various values of $x$ in Wu-Zhang system between MADM and MVIM [17] when

$$
b_{0}=k_{1}=0.1, k_{2}=k_{3}=0.01, t=5, \text { and } y=20
$$

\begin{tabular}{|c|c|c|c|c|}
\hline$x$ & $v_{\text {exact }}$ & $v_{\text {MADM }}$ & $\left|v_{\text {exact }}-v_{\text {MVIM }}\right|$ & $\left|v_{\text {exact }}-v_{\text {MADM }}\right|$ \\
\hline-50 & 0.0884547231 & 0.884547231 & $8.08571164 \mathrm{E}-9$ & $1.039242830 \mathrm{E}-11$ \\
\hline-40 & 0.0884657604 & 0.0884657604 & $5.96388798 \mathrm{E}-8$ & $6.330797530 \mathrm{E}-11$ \\
\hline-30 & 0.0885469904 & 0.0885469900 & $4.34887678 \mathrm{E}-7$ & $3.394419403 \mathrm{E}-10$ \\
\hline-20 & 0.0891299315 & 0.0891299291 & $2.91626141 \mathrm{E}-6$ & $2.438538578 \mathrm{E}-9$ \\
\hline-10 & 0.0926659316 & 0.0926659213 & $1.07971370 \mathrm{E}-5$ & $1.027749896 \mathrm{E}-8$ \\
\hline 0 & 0.102828077 & 0.102828107 & $5.87519681 \mathrm{E}-6$ & $3.050061683 \mathrm{E}-8$ \\
\hline 10 & 0.109795135 & 0.109795126 & $7.18185473 \mathrm{E}-6$ & $8.962341521 \mathrm{E}-9$ \\
\hline 20 & 0.111293272 & 0.111293273 & $1.30807366 \mathrm{E}-6$ & $5.392973059 \mathrm{E}-10$ \\
\hline 30 & 0.111512337 & 0.111512337 & $1.84431598 \mathrm{E}-7$ & $1.072972068 \mathrm{E}-10$ \\
\hline 40 & 0.111542307 & 0.111542307 & $2.50992772 \mathrm{E}-8$ & $2.078596792 \mathrm{E}-11$ \\
\hline 50 & 0.111546369 & 0.111546369 & $3.39937593 \mathrm{E}-9$ & $5.196177359 \mathrm{E}-12$ \\
\hline MSE & & & $1.93743805 \mathrm{E}-11$ & $1.018378476 \mathrm{E}-16$ \\
\hline
\end{tabular}

Table 3: Comparison of absolute errors using $w_{3}$ for various values of $x$ in Wu-Zhang system between MADM and MVIM [17] when

$$
b_{0}=k_{1}=0.1, k_{2}=k_{3}=0.01, t=5, \text { and } y=20
$$

\begin{tabular}{|c|c|c|c|c|}
\hline$x$ & $w_{\text {exact }}$ & $w_{\text {MADM }}$ & $\left|w_{\text {exact }}-w_{\text {MVIM }}\right|$ & $\left|w_{\text {exact }}-w_{\text {MADM }}\right|$ \\
\hline-50 & $2.01570751 \mathrm{E}-6$ & $2.01569976 \mathrm{E}-6$ & $3.705249242 \mathrm{E}-7$ & $7.749880486 \mathrm{E}-12$ \\
\hline-40 & $1.448799414 \mathrm{E}-5$ & $1.48798843 \mathrm{E}-5$ & $2.73 \mathrm{I} 8146111 \mathrm{E}-6$ & $5.708491389 \mathrm{E}-11$ \\
\hline-30 & $1.09176198 \mathrm{E}-4$ & $1.09175786 \mathrm{E}-4$ & $1.986047907 \mathrm{E}-5$ & $4.118114838 \mathrm{E}-10$ \\
\hline-20 & $7.66334776 \mathrm{E}-4$ & $7.66332142 \mathrm{E}-4$ & $1.302774181 \mathrm{E}-4$ & $2.634558628 \mathrm{E}-9$ \\
\hline-10 & $4.01701111 \mathrm{E}-3$ & $4.01701120 \mathrm{E}-3$ & $4.145730832 \mathrm{E}-4$ & $8.8787905 \mathrm{E}-11$ \\
\hline 0 & $6.32943331 \mathrm{E}-3$ & $6.32939492 \mathrm{E}-3$ & $1.153652836 \mathrm{E}-4$ & $3.839847338 \mathrm{E}-8$ \\
\hline 10 & $1.88812677 \mathrm{E}-3$ & $1.88814161 \mathrm{E}-3$ & $2.265746793 \mathrm{E}-5$ & $1.484359887 \mathrm{E}-8$ \\
\hline 20 & $2.92663787 \mathrm{E}-4$ & $2.92663846 \mathrm{E}-4$ & $6.972254850 \mathrm{E}-7$ & $5.892963187 \mathrm{E}-11$ \\
\hline 30 & $4.03711466 \mathrm{E}-5$ & $4.03709936 \mathrm{E}-5$ & $1.991078199 \mathrm{E}-7$ & $1.529342660 \mathrm{E}-10$ \\
\hline 40 & $5.47785204 \mathrm{E}-6$ & $5.47782786 \mathrm{E}-6$ & $2.87710754 \mathrm{E}-8$ & $2.418224902 \mathrm{E}-11$ \\
\hline 50 & $7.41607526 \mathrm{E}-7$ & $7.41604188 \mathrm{E}-7$ & $3.95110044 \mathrm{E}-9$ & $3.337771675 \mathrm{E}-12$ \\
\hline MSE & & & $1.84607383 \mathrm{E}-8$ & $1.547236655 \mathrm{E}-16$ \\
\hline
\end{tabular}




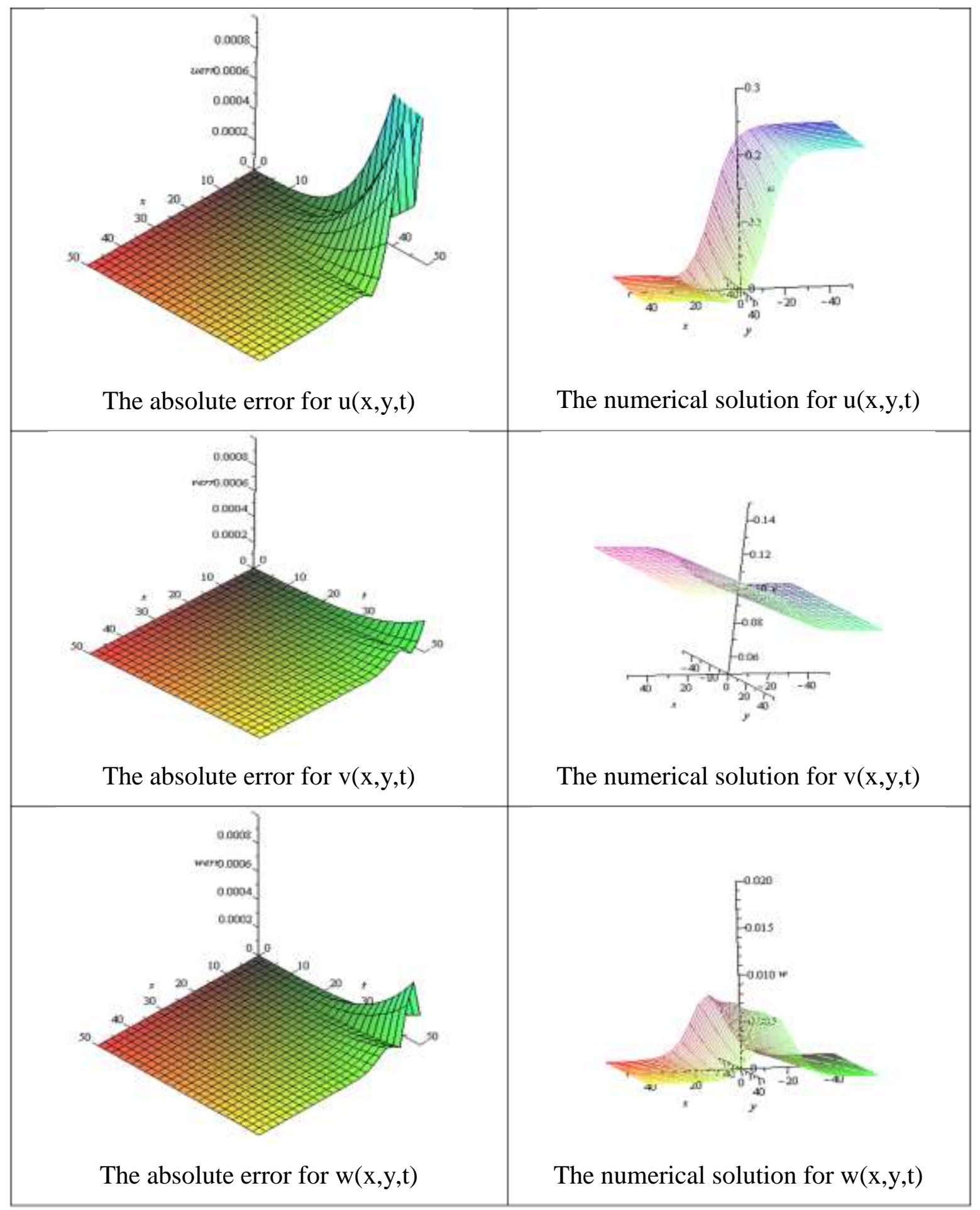

Figure 1: The absolute error for the first three approximation between MADM and the exact solution, for various values of $x$ and $y$ when

$$
b_{0}=0.1, k_{1}=0.1, k_{2}=0.01, k_{3}=0.01 \text {, and } t=5 \text {. }
$$


Table 4: Comparison of absolute errors and mean square error using $u_{3}$ for Wu-Zhang system between MADM with HPM [13] and RDTM [15], for various values of $x$ and $y$ when

$$
b_{0}=0.1, k_{1}=0.05, k_{2}=0.1, k_{3}=0.3 \text {, and } t=2.5
$$

\begin{tabular}{|c|c|c|c|c|}
\hline$(x, y)$ & $u_{\text {exact }}$ & $u_{M A D M}$ & $\begin{array}{c}\left|u_{\text {exact }}-u_{H P M}\right| \\
\left|u_{\text {exact }}-u_{M V I M}\right|\end{array}$ & $\left|u_{\text {exact }}-u_{\text {MADM }}\right|$ \\
\hline$(0.001,-40)$ & $6.25756167 \mathrm{E}-0$ & $6.25757298 \mathrm{E}-0$ & $1.13049917 \mathrm{E}-5$ & $1.13094127 \mathrm{E}-5$ \\
\hline$(0.002,-30)$ & $6.25646611 \mathrm{E}-0$ & $6.25654557 \mathrm{E}-0$ & $7.92908229 \mathrm{E}-5$ & $7.94573088 \mathrm{E}-5$ \\
\hline$(0.003,-20)$ & $6.24897325 \mathrm{E}-0$ & $6.24936663 \mathrm{E}-0$ & $3.85696522 \mathrm{E}-4$ & $3.93378218 \mathrm{E}-4$ \\
\hline$(0.004,-10)$ & $6.21412953 \mathrm{E}-0$ & $6.21299676 \mathrm{E}-0$ & $1.26708625 \mathrm{E}-3$ & $1.13277326 \mathrm{E}-3$ \\
\hline$(0.005,0)$ & $6.16332105 \mathrm{E}-0$ & $6.16444944 \mathrm{E}-0$ & $1.49035435 \mathrm{E}-3$ & $1.12839583 \mathrm{E}-3$ \\
\hline$(0.006,10)$ & $6.14564769 \mathrm{E}-0$ & $6.14581428 \mathrm{E}-0$ & $5.73725768 \mathrm{E}-5$ & $1.66595217 \mathrm{E}-4$ \\
\hline$(0.007,20)$ & $6.14273462 \mathrm{E}-0$ & $6.14247428 \mathrm{E}-0$ & $2.65974103 \mathrm{E}-4$ & $2.60340360 \mathrm{E}-4$ \\
\hline$(0.008,30)$ & $6.14232875 \mathrm{E}-0$ & $6.14228437 \mathrm{E}-0$ & $4.45040837 \mathrm{E}-5$ & $4.43855200 \mathrm{E}-5$ \\
\hline$(0.009,40)$ & $6.14227361 \mathrm{E}-0$ & $6.14226742 \mathrm{E}-0$ & $6.19002050 \mathrm{E}-6$ & $6.18845786 \mathrm{E}-6$ \\
\hline$(0.01,50)$ & $6.14226614 \mathrm{E}-0$ & $6.14226530 \mathrm{E}-0$ & $8.41789424 \mathrm{E}-7$ & $8.41763456 \mathrm{E}-7$ \\
\hline MSE & & & $4.057899493 \mathrm{E}-7$ & $2.815176595 \mathrm{E}-7$ \\
\hline
\end{tabular}

Table 5: Comparison of absolute errors and mean square error using $v_{3}$ for $\mathrm{Wu}$-Zhang system between MADM with HPM [13] and RDTM [15], for various values of $x$ and $y$ when $b_{0}=0.1, k_{1}=0.05, k_{2}=0.1, k_{3}=0.3$, and $t=2.5$

\begin{tabular}{|c|c|c|c|c|}
\hline$(x, y)$ & $v_{\text {exact }}$ & $v_{\text {MADM }}$ & $\begin{array}{c}\left|v_{\text {exact }}-v_{H P M}\right| \\
\left|v_{\text {exact }}-v_{\text {MVIM }}\right|\end{array}$ & $\left|v_{\text {exact }}-v_{\text {MADM }}\right|$ \\
\hline$(0.001,-40)$ & $1.51233361 \mathrm{E}-2$ & $1.51459522 \mathrm{E}-2$ & $2.26099488 \mathrm{E}-5$ & $2.2616027 \mathrm{E}-5$ \\
\hline$(0.002,-30)$ & $1.29322262 \mathrm{E}-2$ & $1.30911405 \mathrm{E}-2$ & $1.58587648 \mathrm{E}-4$ & $1.58914298 \mathrm{E}-4$ \\
\hline$(0.003,-20)$ & $2.05349994 \mathrm{E}-3$ & $1.26674403 \mathrm{E}-3$ & $7.71401056 \mathrm{E}-4$ & $7.86755912 \mathrm{E}-4$ \\
\hline$(0.004,-10)$ & $7.17409386 \mathrm{E}-2$ & $7.40064832 \mathrm{E}-2$ & $2.53417253 \mathrm{E}-3$ & $2.26554453 \mathrm{E}-3$ \\
\hline$(0.005,0)$ & $1.73357903 \mathrm{E}-1$ & $1.71101111 \mathrm{E}-1$ & $2.98071071 \mathrm{E}-3$ & $2.25679205 \mathrm{E}-3$ \\
\hline$(0.006,10)$ & $2.08704626 \mathrm{E}-1$ & $2.08371441 \mathrm{E}-1$ & $1.14747188 \mathrm{E}-4$ & $3.33185019 \mathrm{E}-4$ \\
\hline$(0.007,20)$ & $2.14530751 \mathrm{E}-1$ & $2.15051429 \mathrm{E}-1$ & $5.31948231 \mathrm{E}-4$ & $5.20677749 \mathrm{E}-4$ \\
\hline$(0.008,30)$ & $2.15342497 \mathrm{E}-1$ & $2.15431265 \mathrm{E}-1$ & $8.90041228 \mathrm{E}-5$ & $8.87677480 \mathrm{E}-5$ \\
\hline$(0.009,40)$ & $2.15452784 \mathrm{E}-1$ & $2.15465160 \mathrm{E}-1$ & $1.23800410 \mathrm{E}-5$ & $1.23756930 \mathrm{E}-5$ \\
\hline$(0.01,50)$ & $2.15467717 \mathrm{E}-1$ & $2.15469398 \mathrm{E}-1$ & $1.68148224 \mathrm{E}-6$ & $1.68139959 \mathrm{E}-6$ \\
\hline MSE & & & $1.62316013 \mathrm{E}-6$ & $1.12607062 \mathrm{E}-6$ \\
\hline
\end{tabular}


Table 6: Comparison of absolute errors and mean square error using $v_{3}$ for $\mathrm{Wu}$-Zhang system between MADM with HPM [13] and RDTM [15], for various values of $x$ and $y$ when $b_{0}=0.1, k_{1}=0.05$,

\begin{tabular}{|c|c|c|c|c|}
\hline \multicolumn{5}{|c|}{$k_{2}=0.1, k_{3}=0.3$, and $t=2.5$} \\
\hline$(0.001,-40)$ & $4.99692720 \mathrm{E}-5$ & $4.67304813 \mathrm{E}-5$ & $\begin{array}{c}\left|w_{\text {exact }}-w_{\text {HPM }}\right| \\
\left|w_{\text {exact }}-w_{\text {MVIM }}\right|\end{array}$ & $\left|w_{\text {exact }}-w_{\text {MADM }}\right|$ \\
\hline$(0.002,-30)$ & $3.62278514 \mathrm{E}-4$ & $3.40688032 \mathrm{E}-4$ & $2.14972816 \mathrm{E}-5$ & $2.15904817 \mathrm{E}-5$ \\
\hline$(0.003,-20)$ & $2.33738529 \mathrm{E}-3$ & $2.28043656 \mathrm{E}-3$ & $5.29010050 \mathrm{E}-5$ & $5.69487325 \mathrm{E}-5$ \\
\hline$(0.004,-10)$ & $7.83422424 \mathrm{E}-3$ & $8.54191973 \mathrm{E}-3$ & $7.31802585 \mathrm{E}-4$ & $7.07695496 \mathrm{E}-4$ \\
\hline$(0.005,0)$ & $4.96996963 \mathrm{E}-3$ & $3.63168325 \mathrm{E}-3$ & $1.32491691 \mathrm{E}-3$ & $1.33828638 \mathrm{E}-3$ \\
\hline$(0.006,10)$ & $9.47898572 \mathrm{E}-4$ & $1.37068310 \mathrm{E}-3$ & $3.99459208 \mathrm{E}-4$ & $4.22784533 \mathrm{E}-4$ \\
\hline$(0.007,20)$ & $1.35025275 \mathrm{E}-4$ & $8.08732586 \mathrm{E}-5$ & $5.71504440 \mathrm{E}-5$ & $5.41520164 \mathrm{E}-5$ \\
\hline$(0.008,30)$ & $1.84010586 \mathrm{E}-5$ & $6.03244902 \mathrm{E}-6$ & $1.24360974 \mathrm{E}-5$ & $1.23686096 \mathrm{E}-5$ \\
\hline$(0.009,40)$ & $2.49244365 \mathrm{E}-6$ & $7.14467502 \mathrm{E}-7$ & $1.77924640 \mathrm{E}-6$ & $1.77797617 \mathrm{E}-6$ \\
\hline$(0.01,50)$ & $3.37325458 \mathrm{E}-7$ & $9.47861898 \mathrm{E}-8$ & $2.42562615 \mathrm{E}-7$ & $2.42539268 \mathrm{E}-7$ \\
\hline $\mathrm{MSE}$ & & & $2.457202663 \mathrm{E}-7$ & $2.477398551 \mathrm{E}-7$ \\
\hline
\end{tabular}

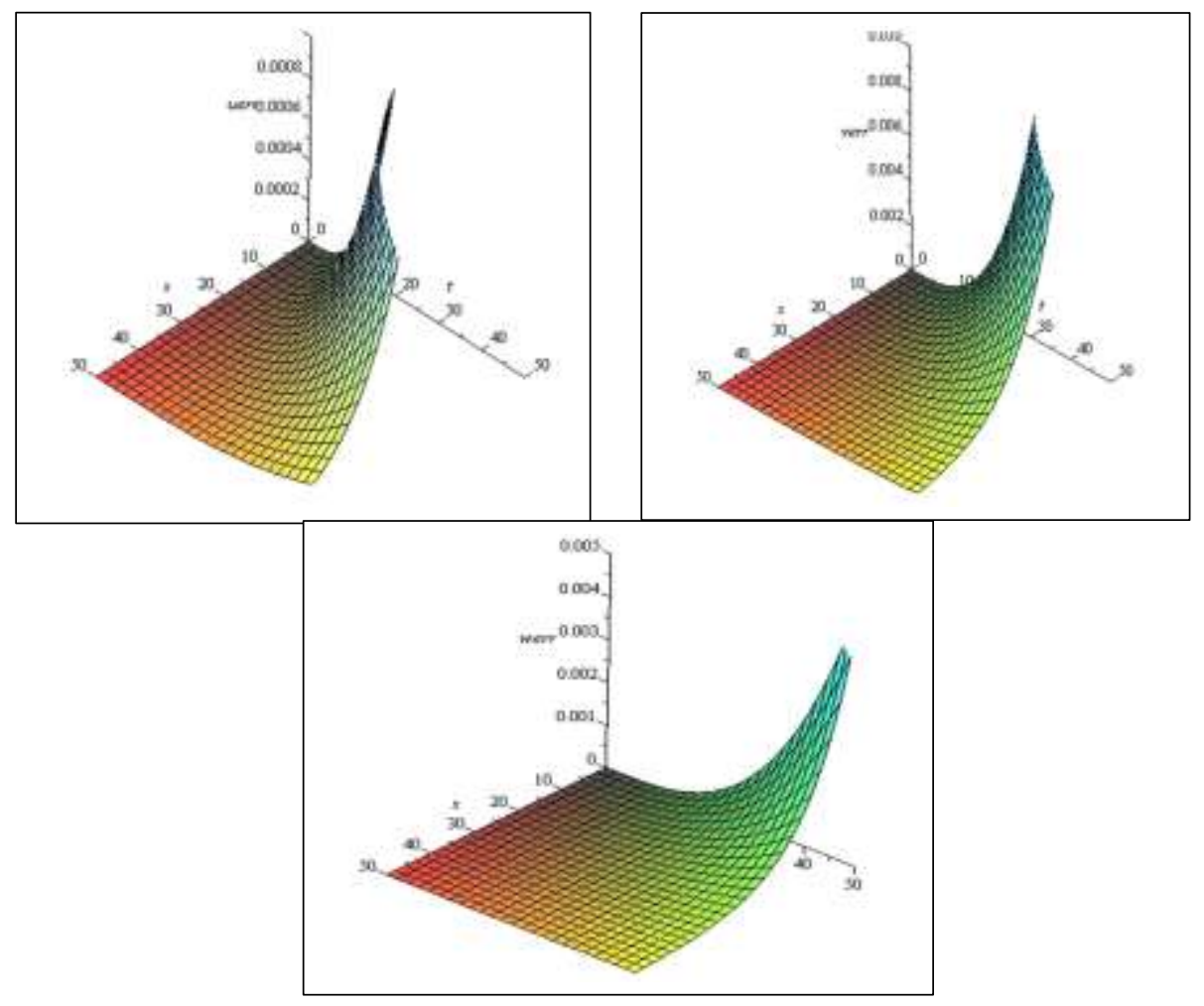

Figure 2: The absolute error for the first three approximation between MADM and the exact solution, for various values of $x$ and $t$ when

$$
b_{0}=0.1, k_{1}=0.05, \quad k_{2}=0.1, k_{3}=0.3, \quad \text { and } y=50
$$




\section{5. conclusions}

The powerful modified adomian decomposition method was employed for analytic treatment of the nonlinear $(2+1)-$ dimensional $\mathrm{Wu}-$ Zhang system, the MADN can be used directly and in a straight forward manner with rapid convergence successive approximations without any restrictive assumptions that may change in the physical behavior of the problem. Comparison at the MADM with several other methods that have been advanced for solving this system, shows that the new technique is reliable, powerful , and promising as shown in the tables (1)-(6). The MADM provides analytic, verifiable, rapidly convergent approximation that yields insight into the character and the behavior of the solution just as in the closed form solution as shown in the figures (1) and (2). All the computations were carried out with the aid of maple 13 software.

\section{REFERENCES}

[1] Adomian, G., (1986), "Nonlinear Stochastic Operator Equations", Academic Press, San Diego, CA.

[2] Al-kresheh , H. A., (2016) "New classes of Adomian polynomials for the Adomian decomposition method ", International Journal of Engineering Science Invention, Volume 5, Issue 3, PP. 37-44.
[3] Al-mazmumy , M., Hendi , F. A., Bakodah , H. O., Alzumi, H. , (2012), "Recent Modifications of Adomian Decomposition

Method for Initial Value Problem in Ordinary Differential Equations", American Journal of Computational Mathematics, 2, pp. 228-234.

[4] Asgari, A., Ganji, D. D., and Davodi, A. G., (2010), "Extended Tanh method and Exp-Function method and its application to (2+1)dimensional dispersive long wave nonlinear equations", journal of the applied mathematics, statistics and informatics (JAMSI), 6, No. 1.

[5] Ayaz, F., (2003), "On the twodimensional differential transform method", Applied Mathematics and Computation, 143:, Pp. 361-374.

[6] Bakodah, H. O., (2012), "Some Modifications of Adomian Decomposition Method Applied to Nonlinear System of Fredholm Integral Equations of the Second Kind ", Int. J. Contemp. Math. Sciences, Vol. 7, No. 19, 929 942.

[7] El-Kalla, I. L., (2007), "Error Analysis of Adomian Series Solution to A Class of Nonlinear Differential Equations", Applied Mathematics E-Notes, 7, pp. 214-221. 
[8] El-Wakil, S. A., and Abdou, M. A., (1998), "New applications of Adomian decomposition method", Chaos Solitons Fractals 33(2), pp. 513-522, 2007.

[9] Fan, E., H. Zhang, (1998), “A note on the homogeneous balance method", Phys. Letters, A246:, Pp. 403-406.

[10] Ganji, D.D., (2006), “The application of He's homotopy perturbation method to nonlinear equations arising in heat transfer", Physics Letters A, A355:, Pp. 337-341.

[11] He, J. H., (2000), “A coupling method of a homotopy technique and a perturbation technique for non-linear problems", International Journal of Non-Linear Mechanics, 35:, Pp. 37-43.

[12] Keskin, Y., G. Oturanc, (2010 ), "Numerical solution of Regularized Long Wave equation by reduced differential transform method", Applied Mathematical Sciences, 4:, Pp. 1221- 1231.

[13] Ma, Z. Y., (2008 ), "Homotopy Perturbation method for the Wu-Zhang equation in fluid dynamics", Journal of physics; conference series 96, 012182.
[14] Mirzazadeh, M., and al., (2017), "Solitons and other solutions to Wu-Zhang system", Nonlinear analysis: modeling and control, Vol. 22, No. 4, Pp. 441-458.

[15] Taghizadeh, N., Akbari, M. and Shahidi, M., (2011), "Application of Reduced Differential Transform method to the Wu-Zhang equation", Australian journal of basic and applied sciences, 5 (5); Pp. 565-571.

[16] Wu, T.Y., J.E. Zhang, (1996), "On modeling nonlinear long wave", in: L.P. Cook, V. Roytbhurd, M. Tulin (Eds.), Mathematics is for Solving Problems, SIAM, pp: 233.

[17] Zayed, E. M. E. and Abdel Rahman, H. M., (2009), “ On solving the Kdv-Burger's equation and the $\mathrm{Wu}$-Zhang equations using the modified variational iteration method", International Journal of Nonlinear Sciences and Numerical Simulation, 10 (9), Pp. 1093-1103. 


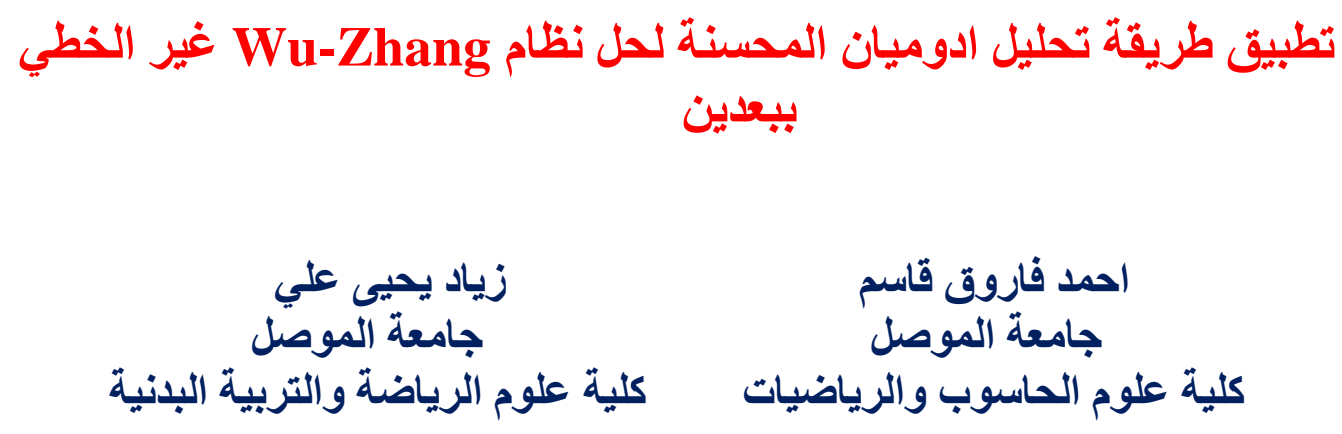

المستخلص

في هذا البحث، طريقة ادرمين الحسنة (MADM) استخدمت لحل نظام WU-Zhang غير الخطي

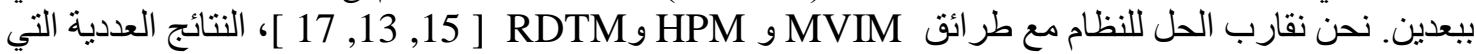

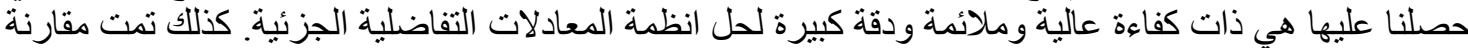
النتائج بين الطريقة المقترحة و الطر ائق الأخرى في بعض كضان الأمثلة لإظهار كفاءة كل طريقة. 